\title{
Expanded CNS Myelin Sheaths Formed In Situ in the Presence of an IgM Antigalactocerebroside-Producing Hybridoma
}

\author{
Jack Rosenbluth, Wei-Lan Liang, Zhiguang Liu, Danzhu Guo, and Rolf Schiff \\ Department of Physiology and Neuroscience and Institute of Rehabilitation Medicine, New York University School of \\ Medicine, New York, New York 10016
}

\begin{abstract}
When 01 hybridoma cells, which produce an IgM antigalactocerebroside, are implanted into the dorsal columns of $4-8 \mathrm{~d}$ rat spinal cord, some of the myelin that subsequently develops in the immediate vicinity displays an abnormal periodicity. The spacings that are seen cluster at $\sim 19 \mathrm{~nm}$ and $31 \mathrm{~nm}$, roughly two and three times the normal $11 \mathrm{~nm}$ spacing. In the expanded sheaths, major dense lines are separated by broad extracellular spaces containing a dense material in which single or double rows of $\sim 10 \mathrm{~nm}$ circular profiles can be identified, consistent with the "central rings" of $\lg M$ molecules. Because $\lg M$ is
\end{abstract}

multivalent, it may serve to link adjacent lamellae together in place of intrinsic myelin molecules that normally interact at close range. Extensive direct contact between myelin components of successive myelin lamellae is thus not essential to signal the growth of the oligodendrocyte membrane or the spiral wrapping of that membrane around axons during myelinogenesis, or to stabilize the myelin spiral that forms.

Key words: demyelination; remyelination; myelinogenesis; multiple sclerosis; IgM gammopathy; paraproteinemia; immunoglobulin $M$; glycolipid; neuropathy
The structure of compact myelin is thought to be stabilized by the interaction of intrinsic myelin components in successive layers with one another. In addition, some of these elements may serve as guideposts during myelin formation (Lemke and Axel, 1985), permitting successive lamellae to recognize and follow those laid down previously.

A recent study of a knockout mouse (Boison et al., 1995) showed, however, that in the absence of at least one major constituent of CNS myelin, proteolipid protein (PLP), which is thought to mediate interlamellar interaction, CNS myelin can form and remain stable, albeit with some abnormality. Continuous intermediate lines were reported to be absent, but focal contacts between successive lamellae were still present, indicating that close interlamellar interaction still occurs in PLP- animals and is presumably mediated by other intrinsic myelin components.

In the current study, we implanted the $\mathrm{O} 1$ hybridoma (Sommer and Schachner, 1981) into the CNS of neonatal rats to explore the role of antimyelin IgM antibodies on myelin formation in vivo. Our results show that an abnormal form of myelin develops in the presence of $\mathrm{O} 1$ that displays a wide separation of lamellae from one another over broad areas, precluding interlamellar interaction. Thus, in such regions, myelin was able to develop without apparent direct contact between lamellae and therefore without direct interaction between the myelin components of successive lamellae. Moreover, the abnormal myelin that formed was stable enough to persist, at least temporarily, indicating that close interlamellar interaction is not required for either the formation or maintenance of the sheath.

Received Dec. 11, 1995; accepted Jan. 22, 1996.

This work was supported by grants from the National Multiple Sclerosis Society and National Institutes of Health. R.S. was supported by National Institutes of Health Training Grant HD 07412 during a part of this study. We thank Ron Morella for expert technical assistance and Dr. Eric Lee, Department of Environmental Medicine, for the statistical analysis.

Correspondence should be addressed to Dr. J. Rosenbluth, RR 714, New York University Medical Center, 400 East 34th Street, New York, NY 10016.

Copyright $(1996$ Society for Neuroscience $0270-6474 / 96 / 162635-07 \$ 05.00 / 0$
In this paper, we consider the manner by which expanded myelin sheaths form in the presence of $\operatorname{IgM}$ and their relevance to the mechanism of myelinogenesis. The physiological consequences of expanded myelin are considered elsewhere (Rosenbluth, et al., 1995a).

A preliminary report of this study has been published previously (Rosenbluth et al., 1995b).

\section{MATERIALS AND METHODS}

Cultures of the $\mathrm{O} 1$ hybridoma, kindly provided by S. Pfeiffer (University of Connecticut), courtesy of I. Sommer (University of Glasgow), were maintained at $36-37^{\circ} \mathrm{C}$ in Falcon flasks in a $\mathrm{CO}_{2}$ incubator by standard methods. For implantation, cells were shaken free, spun down, and resuspended in L-15 medium at a concentration of $\sim 10^{6}-10^{8}$ cells $/ \mathrm{mm}^{3}$. Only live cells were counted (identified by trypan blue exclusion).

Rat pups at ages $\mathrm{P} 4-8$ were anesthetized by hypothermia $\left(-20^{\circ} \mathrm{C}\right.$ for 5-10 min). Laminectomy was performed in the lower thoracic region, and the dorsal spinal cord was exposed; $0.01 \mathrm{ml}$ of the cell suspension was injected into the dorsal columns through a 28 gauge needle, and the skin was closed over the operative site. Cyclosporine $(10 \mathrm{mg} / \mathrm{kg} / \mathrm{d}$, i.p.) was administered beginning on the day of operation. After surgery, animals were watched until they awakened and then were returned to the maternal cage. Six to eighteen days later, animals were reanesthetized and fixed by vascular perfusion with a mixture of $3 \%$ glutaraldehyde and $2 \%$ formaldehyde $(3+2)$ in $0.1 \mathrm{M}$ cacodylate buffer or with buffered $4 \%$ formaldehyde alone, followed by $3+2$ after dissection. Spinal cords were removed, sliced, rinsed, postfixed in 1-2\% osmium tetroxide, dehydrated, and sectioned by standard methods for examination by light and electron microscopy.

Equivalent experiments were carried out using a control hybridoma, CRL 8018 (ATCC), which produces an IgM monoclonal antibody to an irrelevant antigen, in place of the $\mathrm{O} 1$ hybridoma. Culture media were characterized using a monoclonal antibody isotyping kit (Boehringer Mannheim, Indianapolis, IN). Both O1 and CRL8018 were found to produce IgMs with $\kappa$ light chains.

Measurements were made on prints enlarged to $300,000 \times$, in which myelin lamellae were cut normally and did not show signs of separation, splitting, or other artifact. Wherever possible, the myelin period was measured across multiple lamellae. Spacings were grouped, and means were calculated within each group. Measurements were weighted according to the number of lamellae measured.

For immunocytochemical analysis, thin sections of specimens prepared 
Figure 1. Distribution of myelin spacings in expanded myelin sheaths. Myelin periods are confined within narrow ranges at three preferred spacings: $11 \mathrm{~nm}, 19 \mathrm{~nm}$, and $31 \mathrm{~nm}$ (means). Measured spacings were assorted into groups, each spanning $1 \mathrm{~nm}$. The ordinate shows the number of examples $(n)$ in each group.

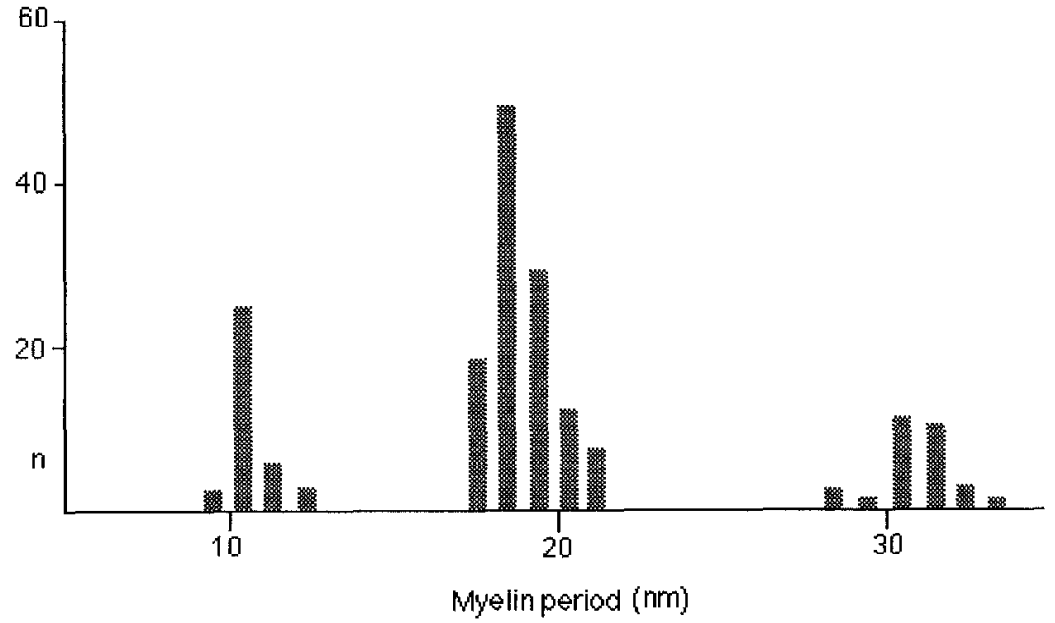

is closely associated with one or the other myelin membrane in an asymmetric manner. Occasional profiles are situated midway between the lamellae, however (Figs. 4, 6D).

In some regions (not used for measurement), the gap is wider still (Figs. 5, 6F), and the intercellular material seems to be divided into three layers: a central une of low density between two moderately dense layers that are directly apposed to the membrane elements and contain the $\sim 10 \mathrm{~nm}$ circular structures described above. The central paler band also contains spotty dense material, including circular profiles, but they occur only sparsely here in comparison with the denser bands on either side (Figs. 5, 6F).

Examination of control spinal cords, into which an irrelevant IgM-producing hybridoma (CRL 8018) had been implanted, did not show cvidence of cxpanded myelin shcaths.

Pooled data from anti-IgM-colloidal gold-stained thin sections (Table 1) showed an eightfold increase in labeling of the expanded myelin sheaths (E) compared with background (B). Statistical analysis showed this difference to be highly significant $(p<$ 0.001 ). In contrast, labeling of normal myelin $(\mathrm{N})$ was not significantly different from background. Presumably IgM in interstitial spaces was washed out during specimen preparation, whereas IgM in the expanded myelin sheaths remained trapped within the myelin coils.

\section{DISCUSSION}

In the present study, we show that an abnormal "expanded" form of CNS myelin can develop even when successive lamellae are separated widely and therefore presumably too far apart to interact via intrinsic myelin components. This result was seen in the presence of an antigalactocerebroside IgM capable of bridging between successive myelin lamellae by virtue of its multivalency. Expanded myelin was not found after implantation of an IgG antigalactocerebroside (Rosenbluth et al., 1994), however, or after implantation of a control hybridoma that secretes an IgM directed against an irrelevant, nonmyelin antigen.

Thus, direct extensive interaction between intrinsic myelin molecules in successive layers is not required for myelin formation. Linkage between successive lamellae, even at a distance much greater than normal, is adequate to support myelinogenesis and to provide some stability to the resulting sheaths. The presence of an irrelevant IgM has no effect on myelinogenesis, but the presence of an antimyelin $\operatorname{IgG}$, capable of binding myelin but not capable of bridging between successive lamellae, blocks myelin formation. width of $\sim 20 \mathrm{~nm}$, within which double layers of $\sim 10$ circular profiles are often discernible. Generally, each such profile as described and mounted on electron microscopy grids were blocked The smallest spacing averages $\sim 11 \mathrm{~nm} \pm 0.7$ (SD), which corresponds to the period of normal CNS myelin. Morphologilines alternating with single intermediate lines (see Fig. 6A).

The second spacing seen (Fig. 3) is approximately double the consists of major dense lines, each flanked by the outer dense laminae of the constituent unit membranes. These paired memwide, containing a material of moderate density, within whi roughly circular profiles compressed into the gap (Figs. 3, 6C) are often discernible.

The third spacing (Fig. 4) is approximately triple the normal period, approximating $31 \mathrm{~nm} \pm 3.3$. In this case, the paired membrane elements are identical to those in the second case but are separated by a band of extracellular dense material with a 

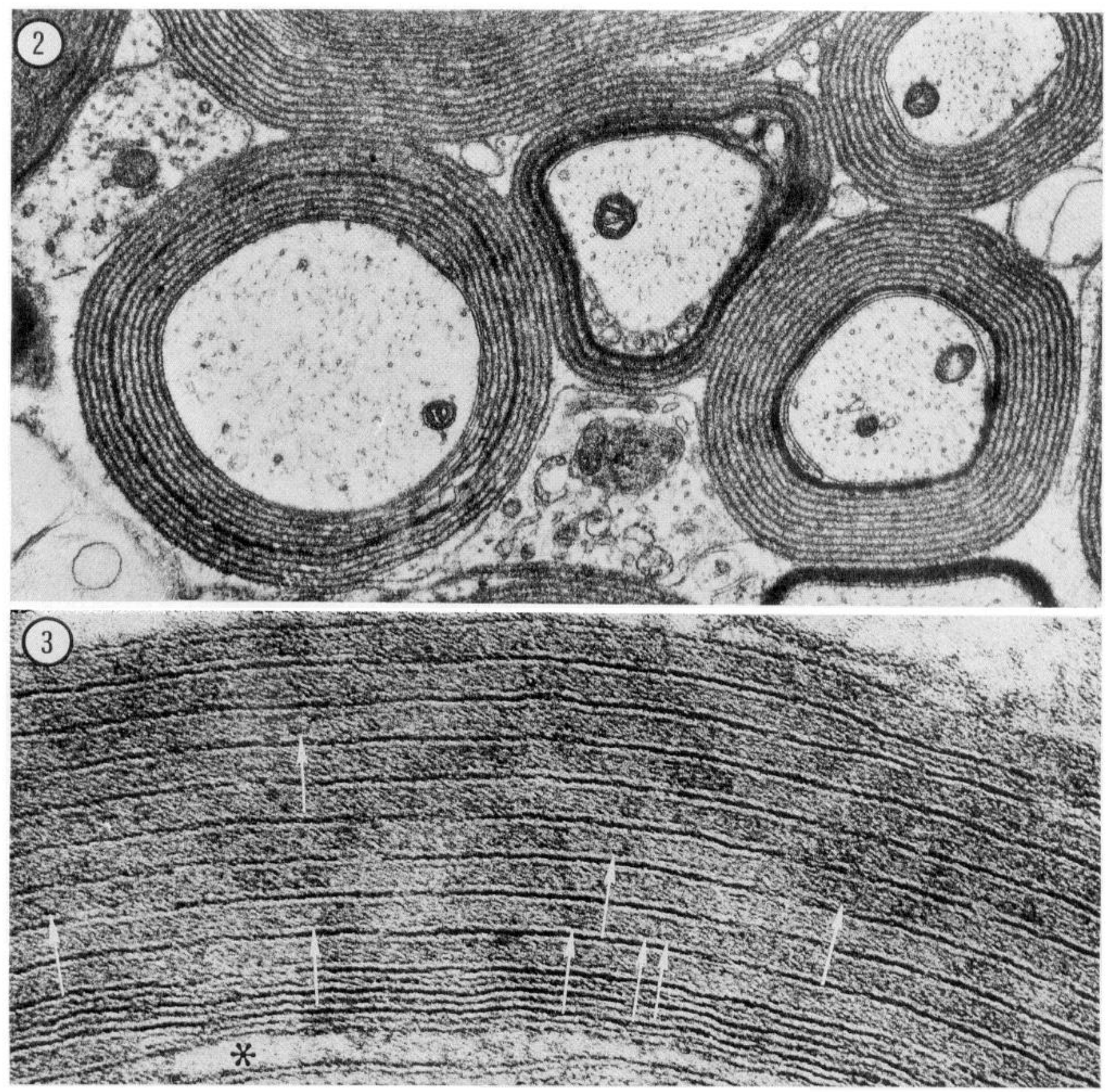

Figure 2. Electron micrograph showing portions of nine myelin sheaths, all of which display expanded myelin, except for the seven-layer sheath at the lower right, which is normally compacted. Magnification: $33,000 \times$

Figure 3. Electron micrograph of a 13-layer expanded myelin sheath. The innermost layer contains cytoplasm (asterisk), and the next three lamellae are normally compacted. The remaining lamellae display an expanded spacing, except for lamellae 11 and 12 , which are nearly apposed at the right. In the region of expanded myelin, the interlamellar spaces are filled with a moderately dense material within which circular profiles are visible (arrows). Magnification: 290,000×.

\section{Incorporation of IgM into myelin}

The presence of a dense material between the lamellae of the expanded myelin sheaths distinguishes these sheaths from those generated by exposure of myelin to hypotonic solutions (Robertson, 1958) and indicates the presence of an intercalated material. A similar interlamellar density is present in the wide-spaced peripheral myelin seen in IgM paraproteinemias (King and Thomas, 1984; Prineas, 1985; Kusunoki et al., 1987; Jacobs and Scadding, 1990; Lach et al., 1993; Latov, 1994) and certain experimental immune diseases of the CNS (Bornstein and Raine, 1976;
Raine et al., 1978) or peripheral nervous system (Raine and Bornstein, 1979; Tatum, 1993). There as well, immunogold studies have demonstrated IgM within the expanded regions of peripheral myelin sheaths (Lach et al., 1993).

How such a large molecule might gain access to the interior recesses of a myelin sheath is unclear. Its size would presumably prevent diffusion along the minuscule extracellular space normally present within an already formed myelin sheath, unless the molecule actively intrudes itself or unless myelin is a much more dynamic structure than is thought. 

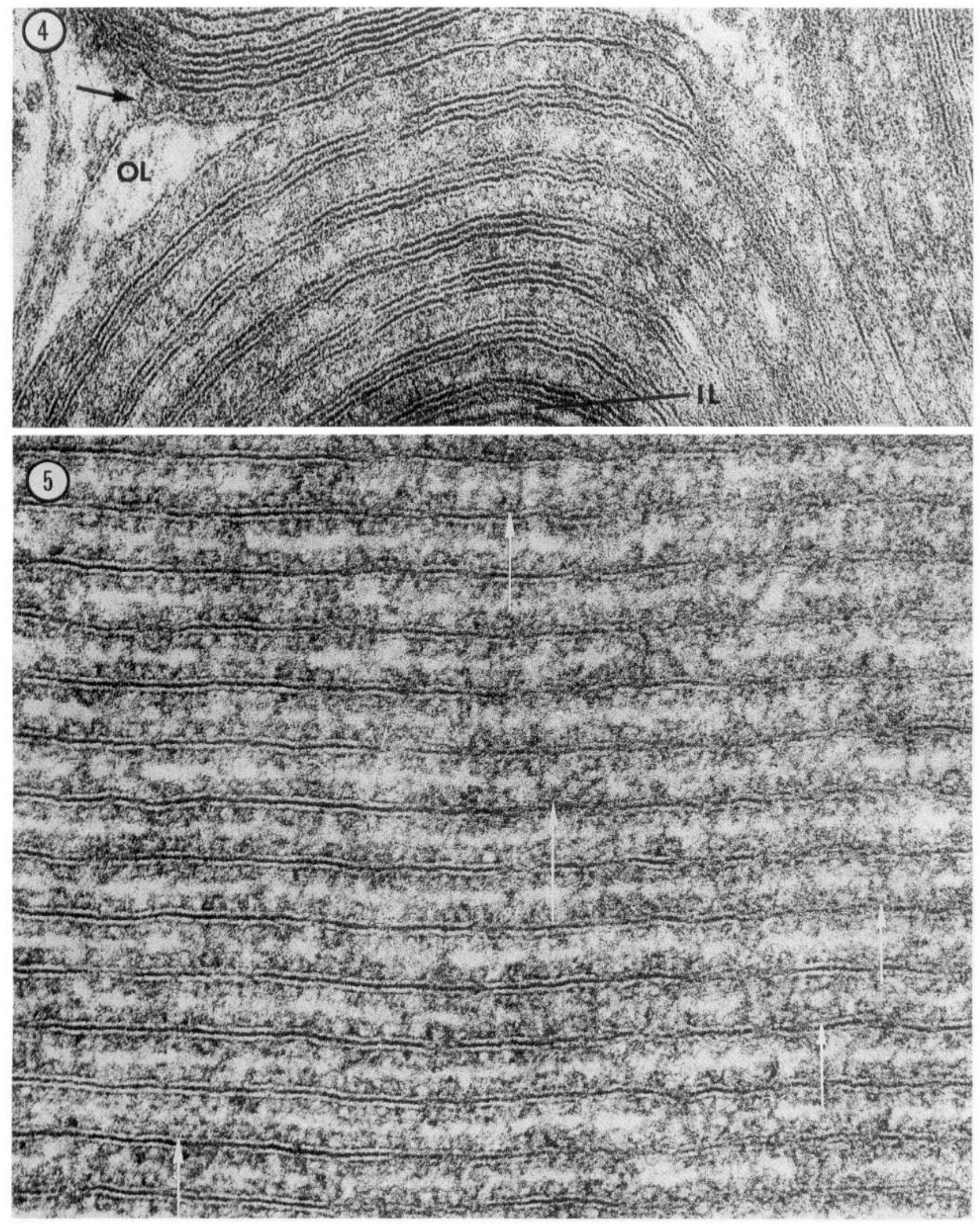

Figure 4. Electron micrograph showing portions of three myelin sheaths. The portion occupying most of the field consists of 13 lamellae, which are alternately separated by wide gaps or intermediate lines. The innermost layer $(I L)$ contains cytoplasm. The second layer is compact and forms an intermediate line with the first layer. The wide spaces between layers $2-3,4-5,6-7,8-9,10-11$, and $12-13$ contain a dense material, most of which seems to consist of circular profiles in double rows. Intermediate lines form between layers $1-2,3-4,5-6,7-8,9-10$, and $11-12$. Dense material also appears between the outermost layer of this sheath $(O L)$ and the sheaths at the upper left and extreme right (arrow). Magnification: $210,000 \times$.

Figure 5. Electron micrograph showing myelin with expanded interlamellar spaces in which rows of circular profiles can be seen attached to each flanking membrane, but are separated from one another by a clear gap. The myelin period here is $\sim 44 \mathrm{~nm}$. In some places, a circular profile is visible within the clear gap midway between the adjacent membranes (arrows). Magnification: 230,000×. 
A

B

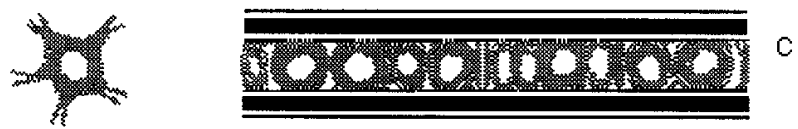

D

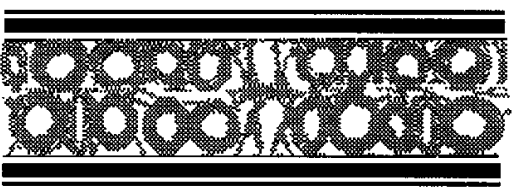

F

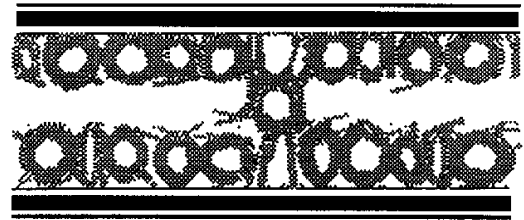

Figure 6. Diagram summarizing proposed models of expanded myelin spacings in relation to $\mathrm{IgM}$ molecules. $A$, Two major dense lines from a region of normal myelin (11 nm period). The intermediate line between them represents the fusion of the outer dense components of the adjacent plasma membranes. $B$, IgM molecule in planar-star conformation composed of a central ring (parallel to the page) and five arms. $C$, Expanded myelin (19 nm period) in which the outer dense components of the adjacent plasma membranes are widely separated by an intervening material resembling a single row of IgM central rings, some of which are seen en face and some rotated around a vertical axis (and therefore foreshortened). The arms are presumably flattened against the membranes or penetrate into them and are thus not visible. (cf. Fig. 3.) D, Expanded myelin $(31 \mathrm{~nm}$ period) in which the adjacent membranes are separated by an intervening material resembling a double row of IgM central rings. Each row is apposed to one or the other membrane. In the center, one IgM is shown midway between the two membranes in a staple conformation, with arms extending toward both membranes. (cf. Fig. 4.) $E$, IgM in the staple conformation. The central ring is perpendicular to the page and appears as a linear bar. Arms extend both up and down. $F$, Expanded myelin (>31 nm period) in which the adjacent membranes are separated by two bands of dense material and a central clear band. This is consistent with two rows of IgM molecules adherent to one or the other membrane, but separated from each other. In the center, one IgM is shown midway between the two membranes in the planar star conformation, with arms extending toward both membranes. (cf. Fig. 5.)

Alternatively, the data presented here suggest that IgM is incorporated as the myelin is formed. O1-coated oligodendroglial processes may thus enwrap axons, carrying the IgM coating along as the myelin spiral is generated. Such expanded myelin sheaths once formed might then permit further movement of $\operatorname{IgM}$ within the widened interlamellar clefts.

\section{Relation of IgM structure to expanded myelin period}

Earlier investigations of isolated $\operatorname{IgM}$ have shown it to display a planar, star-shaped structure, composed of a central Fc ring or disc, - $85-100 \mathrm{~A}$ in diameter, from which five Fab arms, $100 \mathrm{~A}$ long, extend (Feinstein and Munn, 1969; Green, 1969; Metzger, 1970; Feinstein et al., 1971, 1986). These arms are clearly flexible, in view of the existence of "staple" conformations of IgM (Feinstein and Munn, 1969) in which the whole molecule, instead of being planar, has arms bent at nearly a right angle to the central ring, in the way that legs project from a table. Bending is presumed to occur at the hinge region located at the junction of the arms with the central ring (Feinstein et al., 1971).
Table 1. Anti-IgM immunogold label over expanded myelin, normal myelin, or background in electron micrographs

$\begin{array}{llll}\text { Expanded } & \text { Normal } \\ \text { myelin } & \text { myelin } & \text { Background } & \text { Ratio of } \\ \text { (E) } & (\mathrm{N}) & \text { (B) } & \text { densities } p\end{array}$

Total gold particles

(P) $17 \quad 3 \quad 15$

Total area $\left(\mathrm{cm}^{2}\right)$

(A)

125

Particle density

$(\mathrm{P} / \mathrm{A}) \times 1000$

13.6

869

8907

$\mathrm{E}$ vs $\mathrm{B}$ (densities)

$N$ vs $B$ (densities)
3.5

1.7
$<0.001$

$2.1 \quad$ n.s.

Labeling density over expanded myelin profiles is significantly greater than background labeling. n.s., Not significant.

Of the spacings found after $\mathrm{O} 1$ implantation, the $11 \mathrm{~nm}$ period represents myelin in which adjacent lamellae seem to contact one another with apparent fusion of the outermost lamellae (Fig. 6A), as is typical of fixed, dehydrated, epoxy-embedded myelin from mannmalian CNS.

The next two larger spacings are reminiscent of the two separations seen previously between flagella linked by IgM antibodies (Feinstein et al., 1971). In that study, a $\sim 20 \mathrm{~nm}$ separation was found when the linking antibody was in the staple conformation, and a $\sim 30 \mathrm{~nm}$ separation was found when the $\operatorname{IgM}$ was in the planar star conformation.

In the expanded myelin sheaths, however, the $\sim 19 \mathrm{~nm}$ spacing results from the presence of an extracellular cleft of only $\sim 8 \mathrm{~nm}$ between successive myelin lamellac, far smallcr than the separations seen between IgM-linked flagella. Moreover, if IgM molecules in the staple form were present within this cleft, and if the inner rings were parallel to the myelin lamellae, the rings should be seen aligned, edge-on, forming an intermediate density midway between the lamellae. What we see instead, however, are circular structures with no evidence of an intermediate density. The circular structures within the extracellular space in the expanded myelin sheaths are thus more consistent with en face views of the central rings alone. The arms may extend into the depths of the myelin membranes or lie compressed and flattened along their length.

Because IgM is multivalent, such intercalated IgM molecules could serve to anchor adjacent myelin lamellae together, in the same way that molecules normally present within myelin lamellae are assumed to do, but across a much greater distance. Indeed, it seems unlikely that the molecules intrinsic to myclin that normally subserve this role could interact over such a great distance. Specific association between the intrinsic myelin components themselves therefore seems not to be necessary for elaboration of the myelin membrane or for spiral wrapping of that membrane around the axon or for stabilization of the resulting spiral.

If in the regions of expanded myelin sheaths displaying a $31 \mathrm{~nm}$ period the lamellae were separated by a single row of planar stars with arms extended, circular structures would be expected in roughly the middle of the cleft. What is seen, however, are double rows of circles, each row closely applied to one or the other myelin membrane. These could again represent en face images of the central rings of IgM molecules, with their arms flattened, interposed in double rows between successive myelin lamellae.

If the respective rows were offset from one another, i.e., in hexagonal array, the separation between the myelin membranes, 
caused by the dimensions of the central rings alone acting as spacers, would approximate 14-17 nm (assuming a ring diameter of $8-10 \mathrm{~nm}$ ). If the rows were aligned, i.e., in square array, the separation would approximate $16-20 \mathrm{~nm}$, roughly comparable to the actual separation of $\sim 20 \mathrm{~nm}$. When two rows of $\operatorname{IgMs}$ are intercalated asymmetrically between successive myelin membranes, each row presumably binds preferentially to the closer membrane. Because the adjacent rows of IgMs would not be expected to bind each other, what then holds the lamellae together? Some IgMs may exist in the staple form midway between adjacent myelin lamellae and extend arms to bind both facing membranes, thus providing a basis for stabilization at the $31 \mathrm{~nm}$ spacing. The central rings of such molecules could be oriented either parallel to the lamellae, as depicted in Fig. $6 D$, or perpendicular, with arms oriented parallel to the lamellae.

In the expanded myelin sheaths that show a period even larger than $31 \mathrm{~nm}$, the pale central band between successive myelin lamellae may represent a plane along which the double layers of IgM molecules have separated. The occasional densities within this pale band resemble planar stars centered in the clefts (Figs. 4, $6 F$ ). Again, these may extend arms to both facing membranes and impart some stability to these sheaths as well. Nevertheless, separation or shear at the interface between rows of IgMs could lead to eventual disintegration of the myelin structure. Because the myelin sheath consists of a continuous membrane spiral, alternation of the normal period with the $31 \mathrm{~nm}$ period, as seen in Fig. 4 , could also impart partial stability to such sheaths.

\section{Effects of other immunoglobulins}

In vicw of the possibility that the formation of expanded myelin in the presence of $\mathrm{O} 1$ could be related to its interaction with galactocerebroside specifically, we also implanted the $\mathrm{O} 4 \mathrm{hy}$ bridoma, which secretes an IgM directed against other constituents of myelin and oligodendrocytes, including sulfatides and seminolipids (Bansal et al., 1989). Wide-spaced sheaths developed in this case as well (Rosenbluth et al., 1995b); however, after implanting a control hybridoma (CRL8018) that secretes an IgM directed against an irrelevant antigen, not located in oligodendrocyte membranes or myelin sheaths, no expanded myelin was found.

Although multivalent IgMs apparently are able to substitute for intrinsic myelin constituents during myelin formation, we have no evidence that $\mathrm{IgG}$ molecules can. Our previous study of the antiGalC hybridoma (Ranscht et al., 1982), which secretes an IgG3, showed failure of myelin formation, resulting in the development of large amyelinated fascicles of axons, but no widespaced myelin (Rosenbluth et al., 1994). One possible explanation for this finding is that IgG present during myelin formation may indeed bind to the oligodendrocyte membrane and sterically prevent the intrinsic myclin molccules of adjacent lamellae from interacting, but without cross-linking adjacent lamellae in the way that IgMs do.

Antibodies bound to oligodendrocyte processes could activate signaling pathways, in view of evidence for morphological changes in the processes of cultured oligodendrocytes after addition of antigalactocerebroside antibodies to the culture medium (Dyer and Benjamins, 1989). However, as suggested above, the mere binding of the antiGalC $\mathrm{IgG}$, which is unable to bridge between lamellae in the way that the O1 IgM can, evidently is insufficient to signal myelin formation.

\section{CONCLUSIONS}

(1) CNS myelin having an expanded period is able to develop in the presence of the $\mathrm{O} 1$ hybridoma, which produces an IgM antigalactocerebroside.

(2) The expanded sheaths display periodicities that cluster preferentially at $19 \mathrm{~nm}$ and $31 \mathrm{~nm}$.

(3) Close apposition between successive myelin lamellae, with direct interaction between intrinsic myelin components, is not essential for elaboration of the oligodendrocyte membrane, for the spiral wrapping of that membrane around the axon, or for the stabilization of the sheath thus formed. Interaction at a distance, mediated by multivalent antimyelin IgM molecules, can be substituted.

(4) The separation between myelin membranes in the $19 \mathrm{~nm}$ and $31 \mathrm{~nm}$ period sheaths is compatible with the dimensions of a single or double row of IgM central rings between the respective membranes. This interpretation is supported by the presence of visible single and double rows of ring-like structures in electron micrographs.

(5) Myelin exhibiting a $19 \mathrm{~nm}$ pcriod, caused by the intercalation of a single row of IgMs, is likely to be relatively stable because each molecule is capable of binding both myelin membranes across the gap. In contrast, intercalation of two or more rows of IgM molecules, resulting in a period $\geq 31 \mathrm{~nm}$, is likely to be unstable because of the lack of binding of the IgMs to one another and the tendency of the rows to separate or shear apart.

(6) These observations provide bases for both stability and instability of CNS myelin formed (or reformed) in the presence of IgM antimyelin antibodies.

\section{REFERENCES}

Bansal R, Warrington AE, Gard AL, Ranscht B, Pfeiffer SE (1989) Multiple and novel specificities of monoclonal antibodies $\mathrm{O} 1, \mathrm{O} 4$, and $\mathrm{RmAb}$ used in the analysis of oligodendrocyte development. J Neurosci Res 24:548-557.

Boison D, Bussow H, D'Urso D, Muller H-W, Stoffel W (1995) Adhesive properties of proteolipid protein are responsible for the compaction of CNS myelin sheaths. J Neurosci 15:5502-5513.

Bornstein MB, Raine CS (1976) The initial structural lesion in seruminduced demyelination in vitro. Lab Invest 35:391-401

Dyer CA, Benjamins JA (1989) Organization of oligodendroglial membrane sheets: II. Galactocerebroside antibody interactions signal changes in cytoskeleton and myelin basic protein. J Neurosci Rcs $24: 212-221$.

Feinstein A, Munn EA (1969) Conformation of the free and antigenbound IgM antibody molecules. Nature 224:1307-1309.

Feinstein A, Munn EA, Richardson NE (1971) The three-dimensional conformation of gammaM and gammaA globulin molecules. Ann NY Acad Sci 190:104-121.

Feinstein A, Richardson N, Taussig MJ (1986) Immunoglobulin flexibility in complement activation. Immunol Today 7:169-174.

Green NM (1969) Electron microscopy of the immunoglobulins. Adv Immunol 11:1-30.

Jacobs JM, Scadding JW (1990) Morphological changes in IgM paraproteinaemic neuropathy. Acta Neuropathol (Berl) 80:77-84.

King RHM, Thomas PK (1984) The occurrence and significance of myelin with unusually large periodicity. Acta Neuropathol (Berl) 63:319-329.

Kusunoki S, Kohriyama T, Pachner AR, Latov N, Yu RK (1987) Neuropathy and IgM paraproteinemia: differential binding of IgM M-proteins to peripheral nerve glycolipids. Neurology 37:1795-1797.

Lach B, Rippstein P, Atack D, Afar DE, Gregor A (1993) Immunoelectron microscopic localization of monoclonal IgM antibodies in gammopathy associated with peripheral demyelinative neuropathy. Acta Neuropathol (Berl) 85:298-307.

Latov N (1994) Antibodies to glycoconjugates in neuropathy and motor neuron disease. Prog Brain Res 101:295-303. 
Lemke G, Axel R (1985) Isolation and sequence of a cDNA encoding the major structural protein of peripheral myelin. Cell 40:501-508.

Metzger H (1970) Structure and function of gammaM macroglobulins. Adv Immunol 12:57-116.

Prineas JC (1985) The neuropathology of multiple sclerosis. In: Handbook of Clinical Neurology, Vol 3 (Koester JC, ed), pp 213-57. Amsterdam: Elsevier.

Raine CS, Bonnstein MB (1979) Experimental allergic neuritis. Ultrastructure of serum-induced myelin aberrations in peripheral nervous system cultures. Lab Invest 40:423-432.

Raine CS, Diaz M, Pakingan M, Bornstein MB (1978) Antiseruminduced dissociation of myelinogenesis in vitro. Lab Invest 38:397-403.

Ranscht B, Clapshaw PA, Price J, Noble M, Seifert W (1982) Development of oligodendrocytes and Schwann cells studied with a monoclonal antibody against galactocerebroside. Proc Natl Acad Sci USA 79:2709-2713.
Robertson JD (1958) Structural alterations in nerve fibers produced by hypotonic and hypertonic solutions. J Biophys Biochem Cytol 4:349-364.

Rosenbluth J, Liang W-L, Liu Z, Guo D, Schiff R (1995a) Paranoda structural abnormalities in rat CNS myelin developing in vivo in the presence of O1 hybridoma cells. J Neurocytol 24:818-824.

Rosenbluth J, Liang W-L, Liu Z, Guo D, Schiff R (1995b) Wide-spaced myelin formation in situ in the presence of IgM-producing hybridomas. Soc Neurosci Abstr 21:814.

Rosenbluth J, Liu Z, Guo D, Schiff R (1994) Inhibition of CNS myelin development in vivo by implantation of anti-GalC hybridoma cells. $\mathbf{J}$ Neurocytol 23:699-707.

Sommer I, Schachner M (1981) Monoclonal antibodies (O1 to O4) to oligodendrocyte cell surfaces: an immunocytochemical study in the central nervous system. Dev Biol 83:311-27.

Tatum AH (1993) Experimental paraprotein neuropathy, demyelination by passive transfer of human IgM anti-myelin-associated glycoprotein. Ann Neurol 33:502-506. 